\title{
Repeat liver surgery by laparoscopy for a malignant recurrence after previous open or laparoscopic resection
}

\author{
Luigi Cioffi ${ }^{1}$, Andrea Belli², Corrado Fantini' ${ }^{1}$, Alberto D'Agostino', Gianluca Russo', \\ Giulio Belli ${ }^{1}$ \\ ${ }^{1}$ Department of General and Hepato-Pancreato-Biliary Surgery, Loreto Nuovo Hospital, 80142 Naples, Italy \\ ${ }^{2}$ Department of Abdominal Oncology, Division of Surgical Oncology, Istituto Nazionale Tumori Fondazione G. Pascale-IRCCS, \\ 80131 Naples, Italy
}

\begin{abstract}
Aim: This paper reported the experience of one center on repeat laparoscopic liver surgery for metastasis and hepatocellular carcinoma $(\mathrm{HCC})$ with a review of the literature. Methods: This retrospective study included 24 patients who underwent laparoscopic re-intervention (hepatic resection and radiofrequency ablation) for recurrent HCC in cirrhosis $(n=17)$ and for recurrent malignant metastases $(n=7)$ after a previous open or laparoscopic procedure. Patients were divided into two groups according to the first surgical approach. Group 1 underwent open resection and laparoscopic procedure (7 patients), and Group 2 underwent laparoscopic resection and laparoscopic procedure (17 patients). Results: Mean operative time for re-intervention was significantly longer for Group 1 (220.14 $\pm 80.06 \mathrm{~min})$ than for Group $2(150 \pm 56.18 \mathrm{~min} ; P=0.001)$, whereas the mean blood loss and mean hospital stay were comparable in both groups. According to Dindo-Clavien classification, overall morbidity ranged between Grade I and IIla and was similar in both groups. Conclusion: This study suggests that repeat laparoscopic surgery for recurrent hepatic malignant diseases in selected patients is a feasible and safe procedure with good short-term outcomes, but further prospective studies are needed to support these results.
\end{abstract}

Key words: Recurrence; repeat liver surgery; resection

Address for correspondence:

Prof. Giulio Belli, Department of General and Hepato-Pancreato-Biliary Surgery, Loreto Nuovo Hospital, Via A. Vespucci, 80142 Naples, Italy. E-mail: giubelli@unina.it

Received: 20-01-2015, Accepted: 09-03-2015

\section{INTRODUCTION}

Current literature reports significant efficacy of repeat hepatectomies in the treatment of recurrent malignant diseases (both primary and secondary) of the liver. ${ }^{[1-5]}$ The improved clinical outcomes after multidisciplinary treatment have led surgeons and oncologists to work on a new challenge - the management of recurrence. In hepatic surgery, the laparoscopic approach is becoming a widely accepted alternative to open approach especially for tumors located on

\begin{tabular}{|l|c|}
\hline \multicolumn{2}{|c|}{ Access this article online } \\
\hline \multirow{2}{*}{ Website: } & Quick Response Code \\
http://www.hrjournal.net/ & \\
\hline & DOI: \\
10.4103/2394-5079.153915 & \\
&
\end{tabular}

anterior segments of the liver. Nevertheless, at the present, few studies have been done on repeat laparoscopic surgery of the liver because of some technical difficulties of repeated interventions, which is even more challenging if carried out by a minimally invasive approach.

We previously published data on laparoscopic re-interventions for hepatocellular carcinoma (HCC) in cirrhotic liver that described peri-operative outcomes, safety, and feasibility of this procedure. ${ }^{[6]}$ In this paper, our experience on repeat laparoscopic liver surgery for malignant primary and secondary diseases with a review of the literature is reported.

\section{METHODS}

Patients and inclusion criteria

From January 2004 to December 2013, 24 patients underwent a laparoscopic re-intervention (hepatic resection 
and radiofrequency ablation [RFA]) for recurrent HCC in cirrhosis $(n=17)$ and for recurrent metastases from colorectal carcinoma $(n=7)$ after a previous open or laparoscopic procedure. The primary surgical interventions were 7 open and 18 laparoscopic procedures (a laparoscopic segmentectomy was associated with a laparoscopic RFA in 1 patient). Details of hepatic procedures are explained in Table 1. A wedge resection (WR) was performed in association with a laparoscopic left hemicolectomy in 1 case of synchronous metastases; a left lateral sectionectomy with an open left hemicolectomy and a cholecystectomy was performed for a similar case.

The inclusion criteria for the laparoscopic re-intervention were: A well-compensated chronic liver disease (Child-Pugh Class A) without signs of severe portal hypertension in cirrhotic patients, a performance status of Karnosky $\geq 70$, an American Society of Anesthesiology status $\leq 3$, either a single HCC $(\leq 5 \mathrm{~cm})$ or 1 or more metastases when located in the anterior hepatic segments (segments II, III, IVb, $\mathrm{V}$ and $\mathrm{VI})$, or a small $(3 \mathrm{~cm})$ deep HCC for laparoscopic RFA in which major hepatectomy is not recommended. No tumor was biopsied pre-operatively.

The patients were divided into two groups according to the first surgical approach [Table 2]. Group 1 underwent open resection (OR) and laparoscopic procedure (7 patients), and Group 2 underwent laparoscopic resection (LR) and laparoscopic procedure (17 patients). Results from the two groups were compared in a retrospective study. Between the two groups, we analyzed and compared operative time for re-intervention, blood loss, hospital stay, post-operative morbidity, and mortality. Data were expressed as mean \pm standard deviation and represented in Table 3. Differences in means between the groups were compared using Student's $t$-test. $P<0.05$ was considered statistically significant. The hospital review board approved this study.

\section{Surgical technique}

The surgical technique for the repeat laparoscopic hepatic resection was described elsewhere. ${ }^{[6-8]}$ In brief, continuous $\mathrm{CO}_{2}$ pneumoperitoneum was induced using access technique

Table 1: Features of hepatic procedures

\begin{tabular}{|c|c|c|c|c|c|c|c|c|}
\hline Patients & $\begin{array}{l}\text { First } \\
\text { approach }\end{array}$ & First procedure & $\begin{array}{l}\text { Recurrence } \\
\text { site }\end{array}$ & $\begin{array}{l}\text { Second } \\
\text { approach }\end{array}$ & $\begin{array}{l}\text { Redo procedure for } \\
\text { recurrence }\end{array}$ & $\begin{array}{l}\text { Operative time } \\
\text { for second } \\
\text { procedure (min) }\end{array}$ & $\begin{array}{l}\text { Type of } \\
\text { lesion } \\
\text { (number) }\end{array}$ & Size $(\mathrm{mm})$ \\
\hline 1 & OR & Subsegmentectomy & II & $\mathrm{LR}$ & Left lateral sectionectomy & 115 & $\mathrm{HCC}(1)$ & 40 \\
\hline 2 & OR & Segmentectomy & III & LR & Left lateral sectionectomy & 100 & $\mathrm{HCC}(1)$ & 38 \\
\hline 3 & OR & Segmentectomy & IV & LR & $\begin{array}{l}\text { Subsegmentectomy } \\
\text { (converted to laparotomy) }\end{array}$ & 120 & $\mathrm{HCC}(1)$ & 38 \\
\hline 4 & OR & Segmentectomy & IV & LR & Subsegmentectomy & 130 & $\mathrm{HCC}(1)$ & 40 \\
\hline 5 & OR & Subsegmentectomy & VIII & L-RFA & L-RFA & 120 & $\mathrm{HCC}(1)$ & 28 \\
\hline 6 & LR & Left lateral sectionectomy & VII-VIII & L-RFA & L-RFA & 100 & $\mathrm{HCC}(1)$ & 33 \\
\hline 7 & LR & Subsegmentectomy & II & LR & Left lateral sectionectomy & 60 & $\mathrm{HCC}(1)$ & 48 \\
\hline 8 & LR & Segmentectomy & IV & LR & Subsegmentectomy & 80 & $\mathrm{HCC}(1)$ & 30 exophityc \\
\hline 9 & LR & Subsegmentectomy & IV & LR & Subsegmentectomy & 60 & $\mathrm{HCC}(1)$ & 48 exophityc \\
\hline 10 & LR & Subsegmentectomy & v & LR & Segmentectomy & 50 & $\mathrm{HCC}(1)$ & 40 \\
\hline 11 & LR & Subsegmentectomy & VIII & L-RFA & L-RFA & 80 & $\mathrm{HCC}(1)$ & 35 \\
\hline 12 & LR & Segmentectomy & $\mathrm{VI}$ & LR & Segmentectomy & 40 & $\mathrm{HCC}(1)$ & 38 \\
\hline 13 & LR & Segmentectomy & ॥ & LR & Left lateral sectionectomy & 80 & $\mathrm{HCC}(1)$ & 45 \\
\hline 14 & LR & Subsegmentectomy & $\| I I I I$ & LR & Left lateral sectionectomy & 65 & $\mathrm{HCC}(1)$ & 38 \\
\hline 15 & LR & Subsegmentectomy & V & LR & Segmentectomy & 60 & $\mathrm{HCC}(1)$ & 45 \\
\hline 16 & LR & Segmentectomy + L-RFA & VII & L-RFA & L-RFA & 50 & $\mathrm{HCC}(1)$ & 28 \\
\hline 17 & LR & Left lateral sectionectomy & $\|$ & LR & Segmentectomy & 50 & $\mathrm{HCC}(1)$ & 35 \\
\hline 18 & LR & WR & IV & LR & WR & 60 & MTX (1) & 28 \\
\hline 19 & LR & WR & III, VI & LR & WR & 100 & $\operatorname{MTX}(3)$ & $10,23,25$ \\
\hline 20 & LR & WR & VI, V & LR & WR & 80 & $\operatorname{MTX}(3)$ & $17,20,27$ \\
\hline 21 & OR & WR & $\mathrm{II}, \mathrm{V}, \mathrm{VI}$ & LR & WR & 120 & $\operatorname{MTX}(3)$ & $10,28,25$ \\
\hline 22 & OR & $\begin{array}{l}\text { Left hemicolectomy + } \\
\text { cholecystectomy + left } \\
\text { lateral sectionectomy }\end{array}$ & IV-V & LR & $\begin{array}{l}\text { Bisegmentectomy } \\
\text { Third procedure: WR }\end{array}$ & 220 & MTX (1) & 76 \\
\hline 23 & LR & $\begin{array}{l}\text { Left hemicolectomy + } \\
\text { WR }\end{array}$ & II, III, IV & LR & $\begin{array}{l}\text { Left lateral sectionectomy } \\
+ \text { WR } \\
\text { Third procedure: WR }\end{array}$ & 240 & MTX (4) & $28,35,30,38$ \\
\hline 24 & LR & WR & $\mathrm{VI}$ & LR & WR & 120 & MTX (1) & 22 \\
\hline
\end{tabular}


Table 2: Perioperative results

\begin{tabular}{lcccc}
\hline & $\begin{array}{c}\text { Group } 1 \\
(\%)\end{array}$ & $\begin{array}{c}\text { Group 2 } \\
(\%)\end{array}$ & $P$ & S/NS \\
\hline $\begin{array}{l}\text { Extensive adhesions } \\
\text { (grade 3-4) }\end{array}$ & $5(71.4)$ & $2(11.7)$ & 0.01 & $\mathrm{~S}$ \\
$\begin{array}{l}\text { Operative time, min } \\
\text { (mean } \pm \text { SD) }\end{array}$ & $220.14 \pm 80.06$ & $150 \pm 56.18$ & 0.03 & $\mathrm{~S}$ \\
Blood loss, mL (mean \pm SD) & $297 \pm 134$ & $272.2 \pm 120$ & 1.0 & $\mathrm{NS}$ \\
Morbidity & $5(29.4)$ & $2(28.5)$ & 1.0 & $\mathrm{NS}$ \\
Grade I atelectasis & 1 & 1 & - & - \\
Grade I ascites & 1 & 0 & - & - \\
Grade II pneumonia & 2 & 0 & - & - \\
Grade II bleeding & 1 & 0 & - & - \\
Grade IIla perforation & 0 & 1 & - & - \\
Mortality & $\mathrm{Nil}$ & $\mathrm{Nil}$ & - & - \\
Conversion & 1 & 0 & - & - \\
\hline
\end{tabular}

S/NS: significant/nonsignificant; SD: standard deviation

Table 3: Classification of adhesions

\begin{tabular}{ll}
\hline Grade & Description of adhesions \\
\hline 0 & None \\
1 & Thin film, divided by blunt dissection \\
2 & Thin vascular, easily divided by sharp dissection \\
3 & Extensive thick vascular, requires division by sharp dissection \\
4 & Dense, bowel at risk of injury with division \\
\hline
\end{tabular}

of open laparoscopy with the Hasson trocar. In some cases, a safe access to the abdominal cavity was carried out by use of a Visiport ${ }^{\circledR}$ (Covidien, Mansfield, MA, USA), opening the abdominal wall layer by layer, after pneumoperitoneum was achieved with a Verres needle.

During the exploratory laparoscopy, parietal and visceral adhesions were dissected. Such adhesions had to be dissected carefully with the use of specific surgical devices without causing any damage to the gastrointestinal tract before obtaining surgical access to the liver. In this phase, the pneumoperitoneum allowed adhesions to become strained to allow more meticulous assessment and lysis of adherences. The Pringle maneuver was prepared for all patients but was performed only in selected cases $(8 / 24)$.

Anatomical resections (segmentectomy, subsegmentctomy of $\mathrm{IVb}$, bisegmentectomy, and left lateral sectionectomy) were performed for treatment of HCC, and WR was performed for liver metastases.

After an extensive adhesiolysis has been performed, staging abdominal laparoscopy and laparoscopic ultrasonography were carried out to confirm the extension of the lesions and their relationships to the vasculature, to visualize their margins inside the parenchyma, and to exclude a widespread peritoneal carcinosis that might hinder the procedure. Laparoscopic transections were performed with a harmonic scalpel (Harmonic Ace Shears ${ }^{\circledR}$; Ethicon, Endo-Surgery, Cincinnati, OH, USA) or with a vessel sealer (Enseal Tissue Sealer ${ }^{\circledR}$; Ethicon, Endo-Surgery, Cincinnati, OH, USA) or (Ligasure ${ }^{\mathrm{TM}}$; Covidien, Mansfield, MA, USA), and was performed with reduced bleeding, due to a reduction of portal inflow of up to $30 \%$ because of the pneumoperitoneum. The resection bed surfaces were treated with a biologic fibrin glue (Tissucol; Baxter, Wien, Österreich), or a hemostatic gel (Floseal; Baxter, Wien, Österreich), or a sealant patch (TachoSil ${ }^{\circledR}$; Takeda, Linz, Österreich) to minimize risk of biliary leak and to ensure hemostasis.

Bipolar electrocoagulation was used for minor bleeding, and larger structures were secured with ties or either multiple absorbable or nonabsorbable clips.

In order to facilitate the maneuver of left lateral sectionectomy, the left hepatic vein was stapled, and the device was introduced through the trocar located on the right of the patient, and then angled toward the left.

\section{Laparoscopic radiofrequency ablation}

A three-trocar configuration was routinely used. A 12-mm port at the umbilicus housed the $30^{\circ}$ laparoscope. After an extensive adhesiolysis has been performed, staging abdominal laparoscopy and laparoscopic ultrasonography were carried out to identify the positions of the lesions.

As previously described, ${ }^{[9]}$ RFA was carried out with multi-electrode 15-gauge radiofrequency probes (RITA Medical Systems, Mountain View, CA, USA). Hook-shaped retractable electrodes were deployed to a maximum diameter of $3 \mathrm{~cm}$. After every electrode had reached a temperature of $100{ }^{\circ} \mathrm{C}$, the ablation was performed in a step-by-step fashion, with a single step lasting approximately 8-10 min. In two patients with a deep HCC, the size of the lesion was slightly larger than that recommended for a standard RFA (35 and $33 \mathrm{~mm}$, respectively). In these two cases, a Pringle maneuver was carried out during laparoscopy causing vascular occlusion to reduce blood flow and to increase the volume of the ablation.

After track ablation, hemostasis of the liver surface was ensured by bipolar electrocoagulation.

\section{RESULTS}

Repeat laparoscopic hepatic procedures were performed in 24 patients: 6 were treated by left lateral sectionectomy ( 1 associated with a WR), 4 by segmentectomy, 4 by subsegmentectomy ( 1 had conversion to laparotomy), 1 by bysegmentectomy associated with a WR, 4 by laparoscopic 
RFA of HCC, and 5 by WR. Two patients were subjected to a third repeat procedure consisting of laparoscopic WR of segment II and VI, respectively, for a second recurrence of liver metastases.

The laparoscopic procedure was successfully completed in 23 cases $(95.9 \%)$. Adhesions were graded by the staff surgeons using the scale presented in Table 3, similar to that used in a multi-center study on adhesion prevention. ${ }^{[10]}$ Grades 3 and 4 adhesions were present in 5 patients (71.4\%) in Group 1 and 2 patients (11.7\%) in the Group 2.

Of the 24 patients, one underwent conversion to laparotomy in Group 1, not because of adhesions but due to inadequate control of the resection margin for a HCC located in segment IV. One patient, receiving a laparoscopic RFA of a HCC of $28 \mathrm{~mm}$ in VII segment after primary intervention of segmentectomy associated with laparoscopic RFA, was subjected to intestinal resection associated with ileostomy to treat peritonitis from intestinal perforation that occurred during laparoscopic RFA.

The mean operative time for re-intervention was significantly longer for Group $1(220.14 \pm 80.06 \mathrm{~min})$ than for Group $2(150 \pm 56.18 \mathrm{~min} ; P=0.001)$, whereas the mean blood loss was comparable in both groups: $297 \pm 134 \mathrm{~mL}$ in Group 1 and $272.2 \pm 120 \mathrm{~mL}$ in Group $2(P>0.05)$. The mean hospital stay was $6.4 \pm 2.5$ days in Group 1 and $5.2 \pm 3$ days in Group $2(P>0.05)$. The resection margins were disease-free in all the patients.

The overall post-operative morbidity and mortality rates were $29.1 \%(7 / 24)$ and $0 \%$, respectively. According to Dindo-Clavien classification, ${ }^{[1]}$ overall morbidity varied between Grades I and IIla. Morbidity rate was $29.4 \%$ in Group 1 and $28.5 \%$ in Group 2. In Group 1, 2 patients had atelectasis treated by physical therapy (Clavien's Grade II), 2 had pneumonia treated by antibiotics (Clavien's Grade II) and 1 had bleeding from one trocar site treated by compression (Clavien's Grade II). In Group 2, 1 patient presented post-operatively with moderate ascites, 1 with atelectasis (Clavien's Grade I) and 1 presented with intestinal perforation that occurred during a laparoscopic RFA, requiring a re-intervention (Clavien's Grade IIIa).

Long-term outcomes in terms of hepatic recurrence have not yet been evaluated.

\section{DISCUSSION}

Recurrence rate for liver malignancy is estimated at $77-100 \%$ for $\mathrm{HCC}^{[12,13]}$ and $60 \%$ for metastasis from colorectal carcinoma. ${ }^{[14]}$ Nevertheless, current data report efficacy of repeat hepatectomies in the treatment of primary or secondary tumors of the liver. ${ }^{[1-5]}$ At present, studies on laparoscopic hepatic re-interventions are limited. Technical difficulties of both repeat hepatectomy and laparoscopic approach have slowed the spread of laparoscopic re-interventions on the liver. Few papers are available on this procedure, and investigations are biased due to the retrospective nature of these studies, and to the time differences between the series of open and laparoscopic interventions [Table 4]..$^{6,15-22]}$

Tsuchiya et al. ${ }^{[20]}$ reported a cohort of 14 patients affected by HCC, who underwent laparoscopic repeat resection after a primary procedure (laparoscopic hepatectomy, RFA, resection of extrahepatic metastasis, or diagnostic assessment). They demonstrated that 2-year survival in patients with intrahepatic recurrence (100\%) is significantly higher than in those with the extrahepatic recurrence (42.9\%).

Indeed, the surgical strategy can be changed, and survival can be impaired because of the presence of concomitant peritoneal recurrence or because of extensive peritoneal adhesiolysis. Biopsies of suspicious lesions are mandatory to identify carcinomatous foci in dense adhesions to treat the extrahepatic recurrence if possible, or to abstain from a surgical procedure.

Shafaee et al.$^{[18]}$ analyzed the experience of laparoscopic repeat liver resection of three institutions recruiting 76 patients (61 with liver metastasis, 3 with HCC, and 12 with benign lesions) divided into two groups according to the first surgical approach. Peri-operative outcomes (in terms of estimated blood loss and intra-operative transfusions) were better in patients with previous LRs than in patient with previous ORs. Furthermore, long-term outcomes in terms of hepatic recurrence and the need for laparoscopic re-interventions were compared with those of open repeat resection in other studies, ${ }^{[1-5]}$ and similar outcomes were observed.

Table 4: Retrospective studies about laparoscopic repeat surgery of the liver

\begin{tabular}{llcl}
\hline Year & Author & Number & Tumor \\
\hline 2009 & Belli et al. ${ }^{[6]}$ & 12 cases & HCC \\
2009 & Liang et al. ${ }^{[15]}$ & 1 case & HCC \\
2010 & Cheung et al. ${ }^{[16]}$ & 1 case & HCC \\
2011 & Hu et al. ${ }^{[1]]}$ & 6 cases & HCC \\
2011 & Shafaee et al. ${ }^{[18]}$ (tri-institutional) & 76 cases & HCC + metastasis \\
2011 & Nakahira et al. ${ }^{[19]}$ & 15 cases & HCC + metastasis \\
2012 & Tsuchiya et al. ${ }^{[20]}$ & 16 cases & HCC \\
2013 & Kanazawa et al. ${ }^{[21]}$ & 40 cases & HCC \\
2014 & Shelat et al. ${ }^{[22]}$ & 19 cases & HCC + metastasis \\
2015 & Cioffi et al. $($ this series) & 24 cases & HCC + metastasis
\end{tabular}

HCC: hepatocellular carcinoma 
Kanazawa et al. ${ }^{[21]}$ reported a series of 40 patients who underwent hepatic repeat resection for HCC. Twenty patients were previously operated with the open approach and 20 with the laparoscopic approach. Intra-operative blood loss and the incidence of post-operative complications and consequently, post-operative hospital stay were significantly lower in the laparoscopy group.

Shelat et al. ${ }^{[22]}$ reported a series of 19 patients who underwent repeat operated in whom peri-operative data of laparoscopic primary and repeated hepatic resection were compared (outcomes from minor and major resections were considered separately). Liver metastases were the most common indication for repeat resections. The operative time and blood loss were both significantly greater in laparoscopic repeat resection, whereas length of stay and complications did not differ between the groups.

In previous papers that reported our experience in repeat surgery for HCC in cirrhotic liver, we highlighted that a minimally invasive approach applied during the first hepatectomy determines minimal post-operative adhesions and faster and safer adhesiolysis in terms of blood loss and risk of visceral injuries. ${ }^{[23]}$ These factors highlight the advantages of the minimally invasive approach in the management of oncological recurrence of selected cirrhotic or metastatic patients.

In our study, patients with HCC on cirrhosis represent the most part of the cases. This is because patients with multiple lesions in recurrent liver metastases are less often selected for a multiple laparoscopic WR. The mean operative time for re-intervention was significantly longer for the group with previous OR, whereas the mean blood loss and the hospital stay were comparable in both groups. The resection margins were disease-free in all the patients.

A good training in laparoscopic adhesiolysis during minimally invasive incisional hernia repair even in cirrhotic patients can accelerate the learning curve in the lysis of hypervascularized adhesions, facilitated by laparoscopic pneumoperitoneum and optical magnification. ${ }^{[24]}$

The only case of severe complication in our study was in a patient previously treated with a LR followed by a laparoscopic RFA for a recurrent HCC. At the time of re-operation, he was affected by severe thrombocytopenia. The need to perform a safe hemostasis by electrocoagulation on the liver surface after extraction of the RFA probe from the hepatic parenchyma induced us to perform a RFA with the laparoscopic approach. During laparoscopy, the presence of a few thin adhesions (grade evaluated: 0-1) induced us to consider the visceral damage not as a specific complication of adhesiolysis per se, or of the re-operation, but a generic adverse event of laparoscopy. Subsequently, we have restricted indications for the laparoscopic approach of RFA that seems to increase morbidity of an otherwise safe procedure.

In conclusion, this study suggests that repeat laparoscopic surgery for recurrent hepatic malignant diseases in selected patients is a feasible and safe procedure with good short-term outcomes, but further prospective studies are needed to support these results.

\section{REFERENCES}

1. Fong Y, Blumgart LH, Cohen A, Fortner J, Brennan MF. Repeat hepatic resections for metastatic colorectal cancer. Ann Surg 1994;220:657-62.

2. Tuttle TM, Curley SA, Roh MS. Repeat hepatic resection as effective treatment of recurrent colorectal liver metastases. Ann Surg Oncol 1997;4:125-30.

3. Petrowsky H, Gonen M, Jarnagin W, Lorenz M, DeMatteo R, Heinrich S, Blumgart L, Fong Y. Second liver resections are safe and effective treatment for recurrent hepatic metastases from colorectal cancer: a bi-institutional analysis. Ann Surg 2002;235:863-71.

4. Ishiguro S, Akasu T, Fujimoto Y, Yamamoto J, Sakamoto Y, Sano T, Shimada K, Kosuge T, Yamamoto S, Fujita S, Moriya Y. Second hepatectomy for recurrent colorectal liver metastasis: analysis of preoperative prognostic factors. Ann Surg Oncol 2006;13:1579-87.

5. Wicherts DA, de Haas RJ, Salloum C, Andreani P, Pascal G, Sotirov D, Adam R, Castaing D, Azoulay D. Repeat hepatectomy for recurrent colorectal metastases. Br J Surg 2013;100:808-18.

6. Belli G1, Cioffi L, Fantini C, D’Agostino A, Russo G, Limongelli P, Belli A. Laparoscopic redo surgery for recurrent hepatocellular carcinoma in cirrhotic patients: feasibility, safety, and results. Surg Endosc 2009;23:1807-11.

7. Belli G, Fantini C, D’Agostino A, Cioffi L, Limongelli P, Russo G, Belli A. Laparoscopic segment VI liver resection using a left lateral decubitus position: a personal modified technique. J Gastrointest Surg 2008;12:2221-6.

8. Belli G, Limongelli P, Russo G, Fantini C, D’Agostino A, Cioffi L, Belli A. Laparoscopic resection of recurrent hepatocellular carcinoma. In: Asbun HJ, Geller DA. ACS Multimedia Atlas of Surgery: Liver Surgery Volume. Woodbury: Ciné-Med; 2014. ACSV4-36.

9. Belli G, D’Agostino A, Fantini C, Cioffi L, Belli A, Russolillo N, Langella S. Laparoscopic radiofrequency ablation combined with laparoscopic liver resection for more than one HCC on cirrhosis. Surg Laparosc Endosc Percutan Tech 2007;17:331-4.

10. Becker JM, Dayton MT, Fazio VW, Beck DE, Stryker SJ, Wexner SD, Wolff BG, Roberts PL, Smith LE, Sweeney SA, Moore M. Prevention of postoperative abdominal adhesions by a sodium hyaluronate-based bioresorbable membrane: a prospective, randomized, double-blind multicenter study. J Am Coll Surg 1996;183:297-306.

11. Dindo D, Demartines N, Clavien PA. Classification of surgical complications: a new proposal with evaluation in a cohort of 6336 patients and results of a survey. Ann Surg 2004;240:205-13.

12. Poon RT, Fan ST, Lo CM, Ng IO, Liu CL, Lam CM, Wong J. Improving survival results after resection of hepatocellular carcinoma: a prospective study of 377 patients over 10 years. Ann Surg 2001;234:63-70.

13. Tang ZY. Hepatocellular carcinoma - cause, treatment and metastasis. World J Gastroenterol 2001;7:445-54.

14. Homayounfar K, Bleckmann A, Conradi LC, Sprenger T, Lorf T, Niessner M, Sahlmann CO, Meller J, Liersch T, Ghadimi BM. Metastatic 
recurrence after complete resection of colorectal liver metastases: impact of surgery and chemotherapy on survival. Int J Colorectal Dis 2013;28:1009-17.

15. Liang X, Cai XJ, Yu H, Wang YF, Liang YL. Second laparoscopic resection for recurrent hepatocellular carcinoma after initial laparoscopic hepatectomy: case report. Chin Med J (Engl) 2009;122:1359-60.

16. Cheung TT, Ng KK, Poon RT, Chan SC, Lo CM, Fan ST. A case of laparoscopic hepatectomy for recurrent hepatocellular carcinoma. World J Gastroenterol 2010;16:526-30.

17. Hu M, Zhao G, Xu D, Liu R. Laparoscopic repeat resection of recurrent hepatocellular carcinoma. World J Surg 2011;35:648-55.

18. Shafaee Z, Kazaryan AM, Marvin MR, Cannon R, Buell JF, Edwin B, Gayet B. Is laparoscopic repeat hepatectomy feasible? A tri-institutional analysis. J Am Coll Surg 2011;212:171-9.

19. Nakahira S, Takeda Y, Kawashima H, Mukai Y, Hamanaka M, Ono H, Uchiyama C, Kanemura T, Takeno A, Suzuki R, Nakata K, Miki H, Okamura S, Kato T, Tamura S. Pure laparoscopic surgery for repeat hepatectomy. Gan To Kagaku Ryoho 2011;38:2478-80.

20. Tsuchiya M, Otsuka Y, Maeda T, Ishii J, Tamura A, Kaneko H. Efficacy of laparoscopic surgery for recurrent hepatocellular carcinoma. Hepatogastroenterology 2012;59:1333-7.
21. Kanazawa A, Tsukamoto T, Shimizu S, Kodai S, Yamamoto S, Yamazoe S, Ohira G, Nakajima T. Laparoscopic liver resection for treating recurrent hepatocellular carcinoma. J Hepatobiliary Pancreat Sci 2013;20:512-7.

22. Shelat VG, Serin K, Samim M, Besselink MG, Al Saati H, Gioia PD, Pearce NW, Abu Hilal M. Outcomes of repeat laparoscopic liver resection compared to the primary resection. World J Surg 2014;38:3175-80.

23. Belli G, Fantini C, D’Agostino A, Cioffi L, Russo G, Belli A, Limongelli P. Laparoendoscopic single site liver resection for recurrent hepatocellular carcinoma in cirrhosis: first technical note. Surg Laparosc Endosc Percutan Tech 2011;21:e166-8.

24. Belli G, D’Agostino A, Fantini C, Cioffi L, Belli A, Russolillo N, Langella S. Laparoscopic incisional and umbilical hernia repair in cirrhotic patients. Surg Laparosc Endosc Percutan Tech 2006;16:330-3.

How to cite this article: Cioffi L, Belli A, Fantini C, D'Agostino A, Russo G, Belli G. Repeat liver surgery by laparoscopy for a malignant recurrence after previous open or laparoscopic resection. Hepatoma Res 2015;1:30-5.

Source of Support: Nil. Conflict of Interest: None declared. 